\title{
Histoire de l'art et Anthropologie, 6 : le modèle, la copie
}

Dijon, 3 février 2012, sous la coordination d'Eliana Magnani et Daniel Russo

\section{Pauline Duclos-Grenier et Daniel Russo}

\section{(2) OpenEdition} Journals

Édition électronique

URL : https://journals.openedition.org/cem/12306

DOI : $10.4000 /$ cem. 12306

ISSN : 1954-3093

Éditeur

Centre d'études médiévales Saint-Germain d'Auxerre

Référence électronique

Pauline Duclos-Grenier et Daniel Russo, "Histoire de l'art et Anthropologie, 6 : le modèle, la copie », Bulletin du centre d'études médiévales d'Auxerre / BUCEMA [En ligne], 16 | 2012, mis en ligne le 10 décembre 2012, consulté le 22 septembre 2022. URL : http://journals.openedition.org/cem/12306 DOI : https://doi.org/10.4000/cem.12306

Ce document a été généré automatiquement le 22 septembre 2022.

\section{cc) (†) (-)}

Creative Commons - Attribution - Pas d'Utilisation Commerciale - Partage dans les Mêmes Conditions 4.0 International - CC BY-NC-SA 4.0

https://creativecommons.org/licenses/by-nc-sa/4.0/ 


\section{Histoire de l'art et Anthropologie, 6 : le modèle, la copie}

Dijon, 3 février 2012, sous la coordination d'Eliana Magnani et Daniel Russo

\section{Pauline Duclos-Grenier et Daniel Russo}

1 Le séminaire interdisciplinaire d'histoire de l'art et d'anthropologie, organisé par Eliana Magnani et Daniel Russo, a pour objet, pour la troisième année consécutive, les rapports complexes entre le modèle et la copie. En faisant appel à différentes disciplines, histoire et iconographie, histoire des arts visuels, codicologie et diplomatique, anthropologie, la réflexion permet de

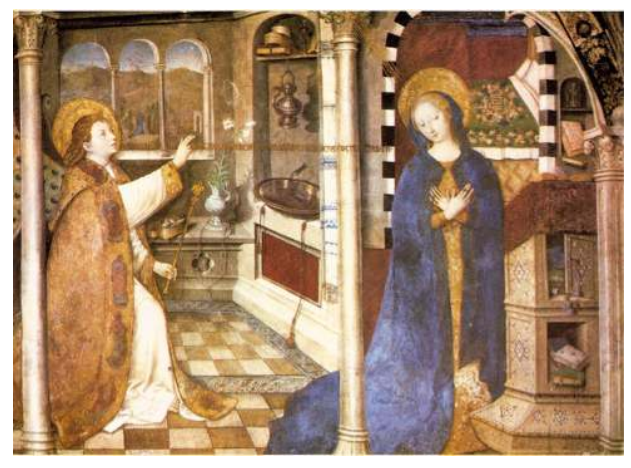
faire émerger diverses modalités de la mise en œuvre d'un processus de fabrication de la copie, que les termes génériques de « modèle » et de " copie » tendent précisément à gommer. Il en va tout pareillement dans les champs actuels de la recherche sur l'invention dans le roman médiéval, par exemple, et les actualisations successives des formes et des figures de création ${ }^{1}$. Aussi, après une analyse lexicographique et théorique, et une deuxième journée consacrée aux processus d'actualisation, d'appropriation et d'appréhension structurant les rapports entre le modèle et la copie, cette séance tend à mettre en lumière les multiples enjeux sociaux, culturels et intellectuels qui sous-tendent les rapports «modèle/ copie $»$.

Intervenants :

- Eliana MAGNANI, Copies figurées d'actes royaux, modèles graphiques et inventions (Bourgogne, $\mathrm{XI}^{\mathrm{e}}$ siècle)

-Anne RITZ-GUILBERT, Copier pour classer au XVII siècle: François-Roger de Gaignières (1642-1715) 
- Séverine FERRARO, L'image et ses modèles; l'image en tant que modèle: l'exemple de l'Annonciation dans l'art mural du Moyen Âge, France et Italie

- Marie CHARBONNEL, Du livre au mur : rituel liturgique, copies de textes bibliques et de prières dans la commande « privée » des décors peints en Auvergne au Moyen Âge

- Daniel Russo, Copie de copies, ou les propriétés de la métaphore architecturale. L'exemple des «tables des canons évangéliques » $\left(\mathrm{VI}^{\mathrm{e}}-\mathrm{XII}^{\mathrm{e}}\right.$ siècle $)$

3 C'est donc par ces idées que Daniel Russo a introduit la séance, rappelant à quel point les disciplines des sciences humaines sont devenues perméables et que le dialogue entre elles est devenu non seulement souhaitable, mais nécessaire. Par ailleurs, le travail de définition et de terminologie auquel se livre ce séminaire est, par conséquent, de plus en plus urgent pour assurer à une démarche interdisciplinaire un socle méthodologique ferme et pour rendre possibles ces échanges ${ }^{2}$.

4 La première intervention de la journée, proposée par Eliana Magnani, a porté sur les copies figurées des actes royaux de la Bourgogne du $\mathrm{XI}^{\mathrm{e}}$ siècle, et la mise en évidence à la fois de modèles graphiques, mais également de l'émergence d'une part d'invention dans les copies de ces modèles. Elle montre comment se mettent en place, par et pour ces documents, une véritable "rhétorique visuelle» et une visualité des documents telles que les définit Peter Rück ${ }^{3}$, comme une production de sens de et par la mise en page même. Il s'agit d'identifier instantanément, de manière presque intuitive, le type du document, mécanisme cognitif qui lit la page tout entière comme un symbole graphique. Aussi, comme toute forme de poétique, tout ce qui relève d'une production graphique fait sens : variation des polices, couleurs, chrismes, forme du parchemin. Il s'agit donc de considérer ces documents également comme des artefacts; comme un ensemble organiquement solidaire, mais également en mouvement; comme un processus où les signes sont faits pour être vus et entendus. Les documents de la diplomatique sont donc désormais considérés comme producteurs de signifiance par leur matérialité même.

5 Suivant cette rapide présentation, la réflexion se décompose en deux temps. Eliana Magnani s'interroge, en premier lieu, sur la manière dont la très longue durée de l'utilisation des signes visuels, depuis l'Antiquité tardive - sceaux et chrismes par exemple -, charge continuellement ces derniers d'une signifiance plus ou moins actualisée selon les époques. Ces récurrences de signes finissent par faire partie intégrante de l'agencement de l'objet, au sens d'agency (Alfred Gell, 1945-1997) et deviennent des attendus du type qu'ils contribuent à faire émerger. Dans un deuxième temps, elle réfléchit à ce que ces éléments graphiques apportent à la compréhension des cartulaires ecclésiastiques, qui contiennent, notamment, des copies d'actes royaux. $\mathrm{Du} \mathrm{XI}{ }^{\mathrm{e}}$ au XIII ${ }^{\mathrm{e}}$ siècle, se dégage dans ces recueils, de manière générale, une tendance à reproduire d'abord ces signes graphiques hérités de l'Antiquité tardive, puis à leur abandon progressif au profit de la lettre initiale, habitée ou enluminée, témoignage d'un changement d'attente vis-à-vis de ces documents. Pourtant, c'est entre la fin du $\mathrm{x}^{\mathrm{e}}$ et le $\mathrm{XI}^{\mathrm{e}}$ siècle que les monastères bourguignons, gagnés par la reformatio, se mettent à produire ce que l'historien pourrait qualifier de pseudo-diplômes mérovingiens et carolingiens.

6 L'étude du rapport entre les actes mérovingiens et carolingiens authentiques et ces diplômes créés a posteriori est riche d'enseignements. Eliana Magnani choisit quelques diplômes royaux - Clotaire III et Thierry III au VII ${ }^{e}$ siècle ; Charles le Chauve au IX ${ }^{e}$ siècle - et en dégage les caractéristiques graphiques principales. De grand format, ces 
documents sont littéralement encadrés par une formule graphique stéréotypée: un chrisme au début du texte, suivi par une ligne de lettres allongées, le corps du texte en modules plus petits, puis une croix ou de nouveau un chrisme en bas du document, auxquels succèdent encore une fois des lettres allongées suivies du monogramme et du sceau. Cet encadrement graphique fonctionne comme un repère visuel.

Les pseudo-diplômes mérovingiens sont, quant à eux, de dimensions plus réduites, adaptées au format des codex. C'est le cas par exemple des diplômes de Clovis et de Clotaire $\mathrm{I}^{\mathrm{er}}$, contenu dans le manuscrit 1 de la bibliothèque de Semur-en-Auxois, qui suivent le livret hagio-liturgique du fondateur et abbé du monastère de Saint-Jean-deRéôme vers $470^{4}$. Le pseudo-diplôme royal de Clovis, qui se présente comme quasi contemporain de la fondation de l'abbaye, place cette dernière sous la protection du roi qui lui assure, par ailleurs, l'immunité ainsi que des sources de revenus. La date restituée est 498 , mais le document a sans doute été fabriqué au XI ${ }^{\mathrm{e}}$ siècle. Cependant, lors de cette composition, une partie du système de signes graphiques des diplômes mérovingiens est réactivée. Il s'agit moins de faire à l'identique pour réaliser un faux, que de faire référence à l'autorité du modèle virtuel, présentifié par son code graphique. On retrouve les lettres allongées de la première ligne, le monogramme; s'ajoutent les différents signes de reconnaissance sous forme de ruches. On retrouve sensiblement le même procédé dans le second diplôme du manuscrit 1 de Semur, dit diplôme de Clotaire I ${ }^{\text {er }}$. L'exemple du pseudo-plaid de Clotaire III en faveur de SaintBénigne de Dijon (fig. 1), en plus du chrisme, reprend également les grandes lettres allongées en début et en fin de texte, mais ménage des espaces laissés vides, destinés à accueillir le sceau ou le monogramme manquant au document ${ }^{5}$.

Fig. 1 - DIJON, Bibliothèque municipale, ms. 591, fol. 69v-70r (Pseudo-plaid de Clotaire III, cartulaire de l'abbaye Saint-Bénigne de Dijon, $\mathrm{XI}^{\mathrm{e}}$ siècle).

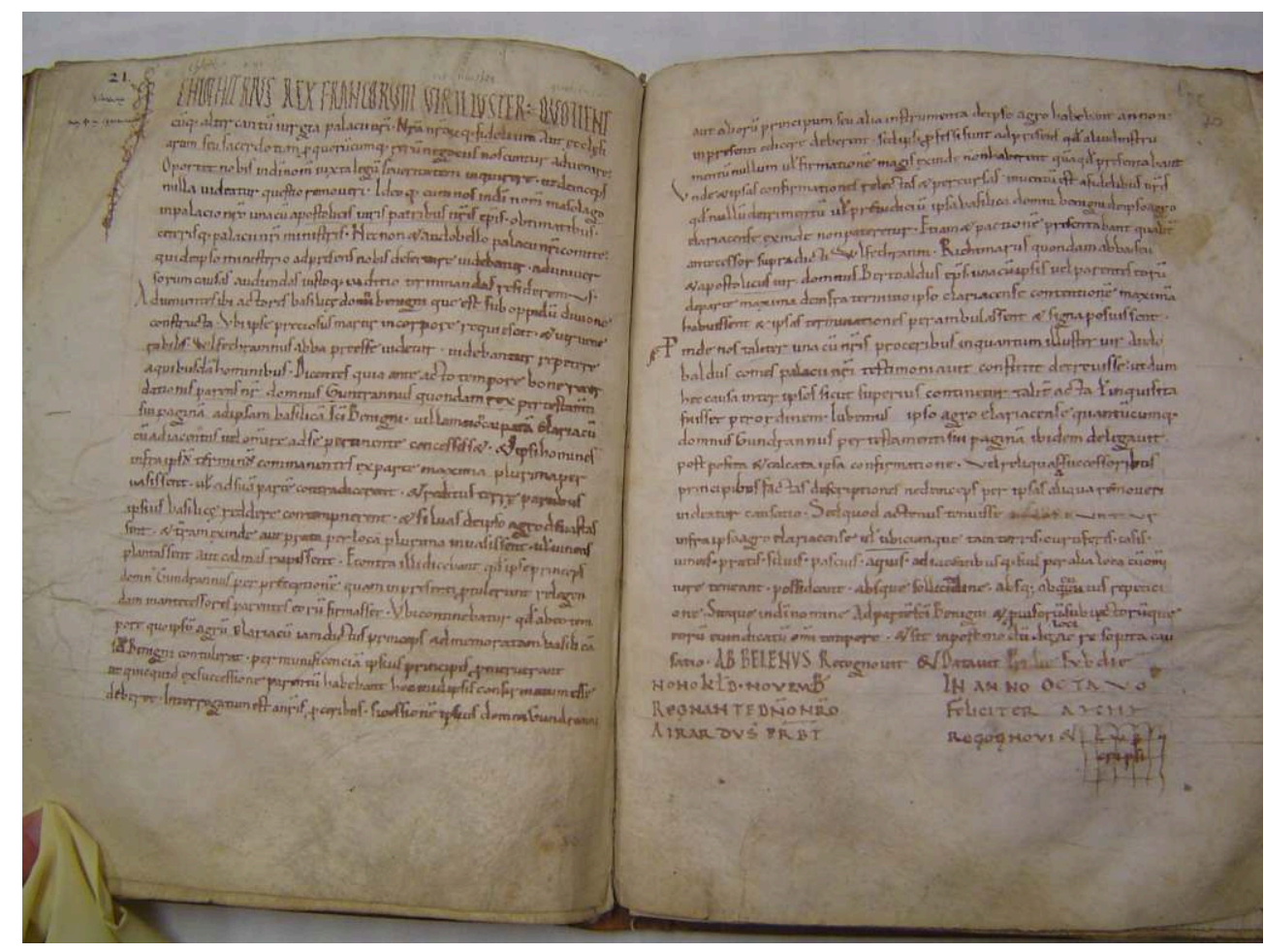



cartulaire » de Saint-Andoche d'Autun, à la fin du $\mathrm{x}^{\mathrm{e}}$ siècle $^{6}$, et les cartulaires A, B et C de Cluny des dernières décennies $\mathrm{du} \mathrm{XI}^{\mathrm{e}}$ siècle ${ }^{7}$ reprennent ces signes, en y ajoutant cependant de la couleur pour attirer davantage le regard.

Ainsi, les signes graphiques hérités de l'Antiquité tardive, et constituant le système visuel des diplômes mérovingiens, sont encore aux $\mathrm{x}^{\mathrm{e}}$-XI $\mathrm{xl}^{\mathrm{e}}$ siècles des attendus nécessaires pour la rhétorique visuelle. Mais le passage de l'acte sur une feuille de parchemin au codex apporte des changements et révèle à la fois la nature de l'enjeu dans la production de sens, conduisant peu à peu à l'abandon de ces signes. Quoi qu'il en soit, il apparait que l'acte de copier ne relève en rien de la reproduction servile et stérile, au contraire, mais témoigne d'une part de création et d'invention, comme d'un jeu avec les codes du système graphique établi, le tout en vue d'une nouvelle production de sens, partagé et compris.

10 L'intervention d'Anne Ritz-Guilbert prolonge la réflexion entamée au sujet de l'archivistique et de la conservation des documents, mais la situe plusieurs siècles en aval, autour de la figure de l'archiviste et collectionneur François-Roger de Gaignières (1642-1715). Ici, le processus de copie n'a pas pour but de produire un système graphique - bien qu'il mette au point un régime de la copie -, mais de permettre un classement d'informations, produisant toutefois également un sens, par le système de classification même qu'il invente. La collection Gaignières rassemble une quantité immense de documents d'archives, de reproductions de monuments de l'époque médiévale, de copies d'inscriptions et d'images de toute sorte. La copie textuelle ou graphique, en de multiples exemplaires, se trouve être le cœur de la collection Gaignières. La masse documentaire ainsi accumulée est répartie entre 2407 volumes, 398 portefeuilles, 2970 volumes imprimés, 1048 tableaux, 4504 médailles.

11 À partir de ce premier état des lieux de la collection, Anne Ritz-Guilbert soulève plusieurs interrogations: si la copie est de toute évidence un moyen de classement, quel est en réalité le projet de Gaignières ? S'agit-il de faire une histoire de France ? Et partant, se pourrait-il que la copie soit pluri-fonctionnelle? Le propos d'Anne RitzGuilbert fera justement émerger une dimension mémorielle de la copie ${ }^{8}$.

Pour ce faire, elle revient sur le problème posé par l'organisation de la collection Gaignières, dont le sens s'est perdu, et qu'elle est cependant parvenue à reconstituer. Le classement opéré par Gaignières est un ensemble de mesures mises en place, au fil de l'accumulation de sa documentation. Le parti pris préalable a été celui de la hiérarchisation des informations. Pour la géographie, il part des cartes générales pour arriver aux cartes à grande échelle; pour l'histoire, il va du général au particulier. Ses recherches permettent à Anne Ritz-Guilbert de recomposer le plan d'ensemble préalablement conçu par Gaignières. Le volume constitue l'unité de ce système. Ce volume se décompose systématiquement en une liste, sorte de plan qui permet de programmer de manière systématique sa recherche documentaire. Il semble ainsi que le classement a précédé l'acquisition de la documentation contenue dans le volume. Les éléments de la liste sont ensuite détaillés. Le reste du matériel utilisé par Gaignières pour son œuvre de classement montre qu'il avait intégré la contrainte de l'inachèvement, propre à la collection à laquelle il se livrait. Il ne travaillait qu'avec des objets "ouverts", cartons, portefeuilles, qui permettaient une insertion continue de documents nouveaux. Au sein des portefeuilles, il laissait également des pages blanches, un feuillet double qui pouvait faire office de petit portefeuille.

Bulletin du centre d'études médiévales d'Auxerre | BUCEMA, 16 | 2012 

copie Gaignières, et que ne copie-t-il pas ? De quels choix intellectuels relève-t-elle et quels processus matériels implique-t-elle? Des actes qu'il copie, Gaignières ne conserve que les noms propres, les dates, les fonctions des individus mentionnés, les sceaux et les signatures. Il garde donc tous les éléments d'authentification en vue de constituer des dossiers prosopographiques. En outre, il indique systématiquement si la copie est intégrale ou partielle. De la même manière, dans le cas d'un relevé de monument, le document visuel fonctionne pour lui comme un document textuel. Gaignières ne dessine pas tout, mais principalement les armes, les épitaphes, les devises. Se dessine un véritable régime de la copie: les noms apparaissent en gras, en modules plus importants que le reste du texte ; les marges recueillent les références aux documents originaux, à gauche, et les dates à droite.

14 L'un des éléments structurants du classement de Gaignières est la multiplicité de la copie. Il peut ainsi classer un même acte en deux endroits différents, reliés par un système de renvoi entre ces deux lieux, ainsi que la localisation du document original. La copie multiple est donc au cœur d'un classement multicritère absolument original pour l'époque. Par ces critères, il met en mouvement et en réseau toute sa collection : son œuvre, sa production de sens, c'est sa collection, telle qu'il l'a conçue.

Anne Ritz-Guilbert remarque, en outre, un phénomène singulier dans le fonctionnement induit par le classement de Gaignières. Ce dernier procède à des copies de portraits, provenant de sources différentes, et qui viennent nourrir un dossier prosopographique, par exemple. On retrouve cependant ces copies de portraits dans le portefeuille consacré aux « modes », mais repris pour en faire des personnages en pied, et ainsi, une galerie de portraits. Il apparaît alors qu'employée dans un autre portefeuille, la copie change, dès lors, de nature et de fonction. appréhendée que dans son intégralité. Si cette intégralité physique a depuis longtemps été pulvérisée, au moins revit-elle sous sa forme théorique au centre de laquelle la copie multifonctionnelle est l'unité de base ${ }^{9}$. de la collection Gaignières. Il s'agit de la copie d'un manuscrit napolitain du XIV siècle de l'ordre du Noud du Saint-Esprit au Droit Désir. Ce manuscrit est très important pour l'histoire des ordres et de la chevalerie, c'est pourquoi Gaignières fait exécuter une copie intégrale en reprenant une structure à l'identique, une copie de l'écriture et des miniatures. Les copies des enluminures ont permis de recréer des figures en pied dans le portefeuille des modes. Quant à la miniature de la première page, elle a été copiée avec un soin inédit dans le classement, au moyen d'un calque retrouvé par ailleurs. Il ne s'agit donc plus uniquement ici d'une copie pour classer, conclut Anne Ritz-Guilbert, mais d'un enjeu mémoriel et patrimonial, de rapport au passé. D'ailleurs, au fil du temps, l'évolution des motivations de Gaignières est perceptible. Si à l'origine sa collection a une vocation documentaire, il propose au début du XVII siècle un tour des provinces pour repérer les monuments à sauvegarder. Il s'agit donc, désormais, également d'une fonction mémorielle de la copie et, par elle, de la collection. Resterait à s'interroger sur ses critères de choix et, entre autres, sur ses rapports avec les milieux de l'érudition locale, par exemple en Bourgogne.

Continuant la discussion entamée sur la dimension pluri-fonctionnelle de la copie au cœur de la collection Gaignières, Daniel Russo s'interroge sur la question du «je » en 
œuvre dans le processus de classement. Ce dernier se révèle en effet " un miroir du je » du sujet Gaignières, comme le théorisait Michel Beaujour dans son ouvrage Miroirs d'encre ${ }^{10}$.

Dans son intervention, Séverine Ferraro questionne justement le rapport réflexif qu'entretient l'image avec la notion de modèle, à travers les représentations de l'Annonciation en France et en Italie. Elle active les rouages de la relation que ces images très codifiées entretiennent avec leurs sources, comprises comme des modèles au sens de sources d'inspiration et, donc, du processus de création de l'image. Elle analyse en retour dans quelle mesure ces images de l'Annonciation, en pleine mutation à la fin du Moyen Âge, tendent de plus en plus à fonctionner elles-mêmes comme des modèles, soulevant ainsi le problème de la réception de ces images selon un processus entraînant d'images qui s'avère, le plus souvent, celui de copies de copies ${ }^{11}$.

Le travail présenté par Séverine Ferraro s'ouvre par l'identification des sources textuelles des images de l'Annonciation. À partir des évangiles canoniques, des évangiles apocryphes et des textes médiévaux - Legenda aurea et Meditationes vitae Christi -, elle isole différentes traditions iconographiques, leurs caractéristiques et leurs aires de diffusion ${ }^{12}$. La tradition orientale, héritée du Protoévangile de Jacques, donne naissance au type de "l'Annonciation à la fontaine ", à celui de «la Vierge filant ", ou encore au type de «l'Incarnation par l'oreille». La tradition occidentale, quant à elle, se fonde essentiellement sur le récit de Luc et se traduit par le type de "la Salutation angélique » par l'archange Gabriel, de « l'Incarnation par le Saint-Esprit » et du Fiat qui témoigne du consentement de la Vierge. Pour chacun des types repérés, elle dégage les codes et les attendus, ces "modèles ", qui participent de la réception et de l'efficacité de l'image. Par exemple, dans les salutations angéliques, les paroles de l'archange sont matérialisées par une inscription, soit libre, soit dans un phylactère. Ces inscriptions créent un double lien très efficace, visuel et textuel, entre les deux personnages. Dans certains cas, en particulier en France, le phylactère est orienté vers le bas. Cette convention exprime l'autorité de la parole énoncée.

Ces modèles iconographiques établis par la tradition, et repris par les artistes tout au long du Moyen Âge, suivent des codes qui évoluent très sensiblement. Ces mutations par rapport aux modèles canoniques entraînent, ou parfois traduisent, un changement de nature dans l'image et de son rapport avec le fidèle. En effet, elle acquiert à la fin du Moyen Âge occidental une dimension dévotionnelle toute nouvelle, en ceci qu'elle se propose désormais elle-même comme un modèle dévotionnel pour le fidèle (fig. 2). Séverine Ferraro identifie trois marqueurs le long de l'évolution. 
Fig. 2 - L'Annonciation, Gênes, église S. Maria di Castello, 1451, Giusto di Ravensburg [source : http://upload.wikimedia.org/wikipedia/commons/d/d3/Giusto_d\%27Alemagnachiesa_santa_maria_di_castello-annunciazione.jpg]

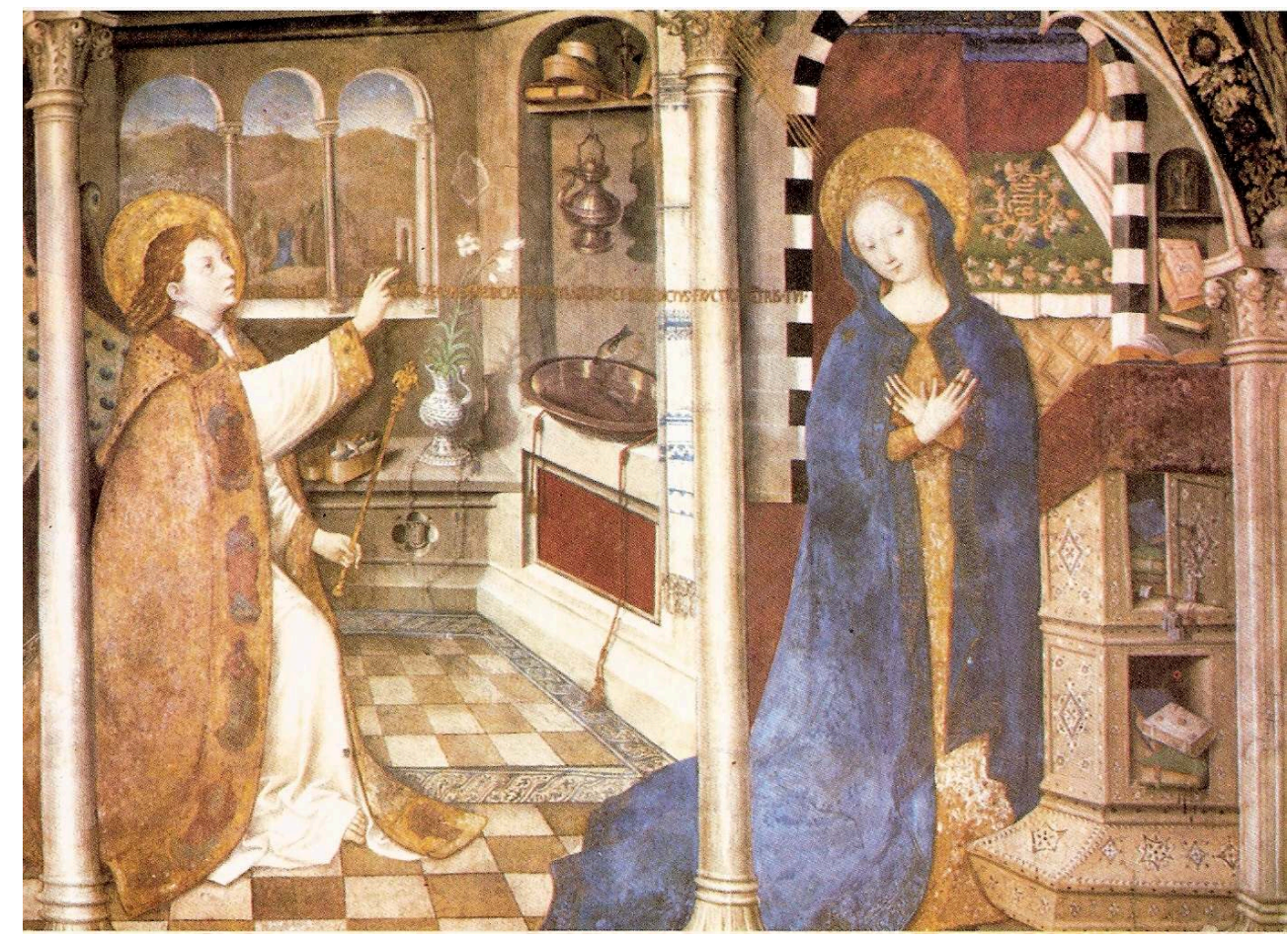

Tout d'abord, les attitudes et la gestuelle des protagonistes, dont la force de « communication non verbale» a été amplement mise en lumière par les travaux de Jean-Claude Schmitt ${ }^{13}$, perdent peu à peu de leur solennité et finissent par s'adoucir, surtout à partir du $\mathrm{XI}^{\mathrm{e}}$ siècle. Elles créent ainsi une plus grande proximité, voire une sympathie, avec les protagonistes et relaient l'évolution spirituelle menée par les ordres mendiants. L'attitude de la Vierge exprime désormais, et de plus en plus souvent, la surprise ou le consentement, celle de Gabriel la bénédiction, geste de la Parole, plus en conformité visuelle avec le contenu de l'Incarnation, ou la révérence ${ }^{14}$. C'est précisément en cela que l'image devient un modèle de dévotion. Sont mis en scène des personnages dont l'attitude est en elle-même un modèle de dévotion, et se trouve choisie comme telle : la Vierge est vue en modèle d'obéissance et de soumission à la volonté divine; pour sa part, Gabriel adopte de plus en plus une gestuelle de vénération, mais aussi de soumission envers la Mère de Dieu. Ce sont des dimensions que souligne la présence, désormais fréquente, des donateurs en train de prier.

Cette thèse de l'image comme modèle est également renforcée par l'apparition d'un type nouveau, celui de la Vierge au livre. Cet objet s'impose dans les derniers siècles du Moyen Âge comme l'attribut-objet de la Vierge, de la sorte mise en scène en plein exercice de sa dévotion personnelle. La peinture d'un prie-Dieu vient occasionnellement renforcer cette dimension d'exercice spirituel recommandé au fidèle.

Enfin, le dernier symptôme de la mutation de l'image de l'Annonciation est la réduction de la salutation angélique à des formules abrégées, tellement signifiantes que seules les abréviations suffisent pour inciter à la récitation complète de l'Ave Maria devant la Vierge ${ }^{15}$. 

étonnants : d'une part, la mutation de la nature de l'image à mesure qu'elle s'affranchit de ses modèles originels; d'autre part, le rapport réflexif dévotionnel que cette nouvelle image propose au « fidèle », dont on attend désormais qu'il « copie » les gestes des «modèles » que sont Gabriel et la Vierge, autrement dit des modèles qui lui sont proposés.

Marie Charbonnel s'intéresse cette fois au processus de la copie de textes bibliques et à leur insertion dans le complexe d'une chapelle privée. Elle réfléchit à la manière dont des inscriptions copiées s'intègrent dans un plus large univers sensoriel ${ }^{16}$, celui en l'occurrence de la chapelle fondée au XIV ${ }^{\mathrm{e}}$ siècle par Gilles Aycelin dans la collégiale Saint-Cerneuf de Billom (Puy-de-Dôme). Elle montre ainsi comment ces inscriptions monumentales, longtemps associées exclusivement au domaine scripturaire, biblique ou liturgique, font en fait également référence à l'oralité, de sorte qu'à la fois le domaine scripturaire et l'oralité fonctionnent comme des sources ou des « modèles » de ces inscriptions ${ }^{17}$. La figure de l'ange joue en outre un rôle clé dans ce système de signes (fig. 3).

Fig. 3 - Ange tenant un phylactère. Billom, collégiale Saint-Cerneuf, Puy-de-Dôme, xIVe siècle (cl. Marie Charbonnel).

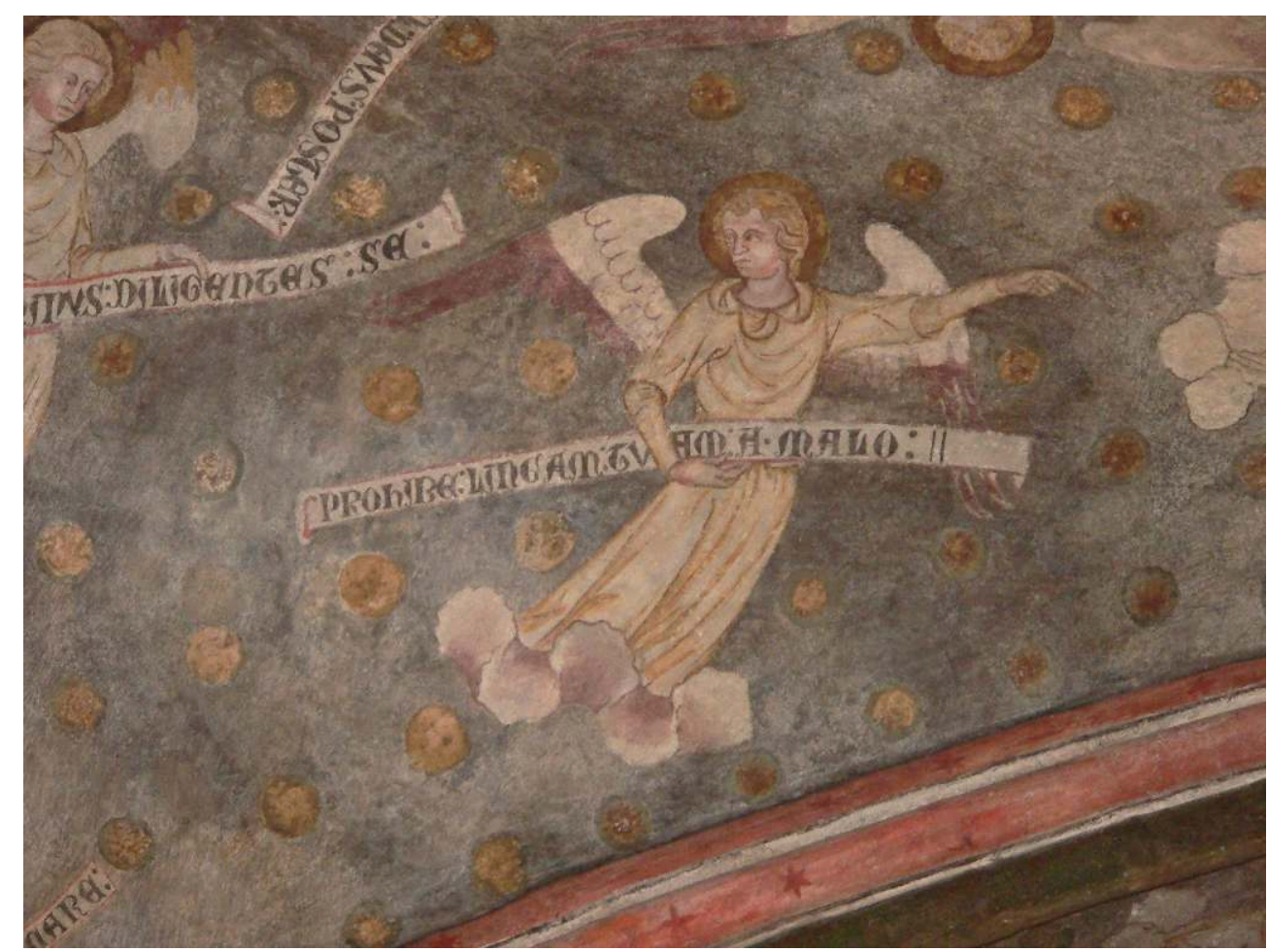

Pour poser les fondements de sa démonstration, Marie Charbonnel présente la chapelle de Saint-Cerneuf, à l'origine indépendante de la collégiale, et notamment l'environnement visuel qu'elle crée. Le programme iconographique est composé de trois registres dont la lecture initie un mouvement ascensionnel. Des prophètes figurent dans le registre inférieur, quand l'Assomption, le Couronnement de la Vierge et l'Ascension occupent le registre supérieur. Le registre médian a, quant à lui, presque intégralement disparu. Marie Charbonnel attire l'attention sur la présence d'anges musiciens aux côtés des scènes mariales. Ils créent un lien, une transition, avec le 
programme iconographique de la voûte peuplée d'anges musiciens dans la travée occidentale et d'anges tenant des phylactères dans la travée orientale. Les quatre évangélistes assurent la liaison entre les deux travées. L'environnement rappelé, Marie Charbonnel soulève la question du sens, de la signifiance de l'inscription des textes des phylactères dans le contexte monumental de cette chapelle. Le rapport entre le lieu, le rituel qui s'y accomplit, les pratiques liturgiques, les images et les chants se trouve condensé dans ces textes apposés sur les phylactères tenus par les anges ${ }^{18}$. Elle montre le mouvement permanent en œuvre dans le complexe monumental entre différents sens, de l'ouïe à la vue, du manuscrit à la pierre.

Les sources textuelles des inscriptions sont le Gloria ainsi que divers psaumes de l'office du lundi ou de la Toussaint, et un ensemble de textes à visée de prescription. Or, ces sources essentiellement exégétiques font l'objet d'une actualisation multiple. Par exemple, ils renvoient autant au champ de l'écrit biblique qu'au champ de l'oralité, oralité par ailleurs rendue omniprésente par les anges musiciens. Ce jeu entre l'écrit et l'oral se retrouve dans d'autres chapelles, comme celle de Beauchamp à Warwick ${ }^{19}$, dans laquelle on retrouve cette association d'anges musiciens et d'anges "phylactophores ». À Billom cependant, certaines inscriptions semblent fonctionner comme une adresse envers la figure du fondateur. Des extraits du Décalogue et du Psaume 33 de saint Augustin, considérés dans les sentences comme les bases de la Loi, pourraient tout autant avoir un rôle dans la quête de la Rédemption qu'être une référence et un hommage à l'œuvre du fondateur, juriste licencié en droit. Quoi qu'il en soit, la lisibilité difficile de ces inscriptions conduit à les interpréter surtout comme la matérialisation du lien entre liturgie terrestre et liturgie céleste, et dont l'ange devient le principal motif iconographique. Plusieurs textes soulignent, en effet, le rôle d'exemplarité endossé par les anges ${ }^{20}$. Leur figuration, leur actualisation dans un complexe ecclésial sont donc des signes visuels et un indice sensoriel très fort des relations tissées entre la voix et la peinture ${ }^{21}$.

L'étude de la chapelle de Billom met en évidence la complexité d'un système de signes très dense entre le lieu (la chapelle, la voûte), la présence angélique qui elle-même crée un lien entre le monde terrestre et le monde céleste, et qui est exprimée soit par l'image, soit par l'épigraphie. S'ajoute la pratique liturgique qui actualise les différents signes, soit par la lecture, soit par le chant, un chant très présent également à travers la figure des anges musiciens et les textes des phylactères, copiés principalement à partir des manuscrits liturgiques. Cet incroyable va-et-vient entre les références, citées et montrées, produit en effet un dense environnement sensoriel au sein d'une trame toute pétrie d'intratextualité.

L'intervention finale de Daniel Russo interroge les propriétés de la métaphore architecturale à travers le processus de la copie, autour de l'exemple des tables des canons évangéliques, depuis le $\mathrm{VI}^{\mathrm{e}}$ siècle jusqu'au $\mathrm{XII}^{\mathrm{e}}$ siècle. Cette métaphore architecturale, utilisée pour peindre les canons des évangiles canoniques, a pour enjeu de présentifier, de rendre présent, l'objet livre lui-même. Elle repose, dès ses origines, sur la création d'un système de lecture classificatoire, comme un modus operandi, celui de la mise en tableau. L'époque carolingienne constitue un moment de mise en forme privilégié d'un type classique de ces tables. La table des canons dans la Bible de SaintBénigne de Dijon est exemplaire du troisième mouvement de l'évolution générale de la reproduction de ces tables dans les bibles, les évangéliaires et autres genres scripturaires. Il s'agit de montrer comment la diffusion d'un modèle, très tôt constitué, 
sert de base à tout un processus de la copie et de mettre en lumière les mécanismes de création de l'image ${ }^{22}$.

31 Les premiers cas retrouvés de mise en place des tables des canons évangéliques datent $\mathrm{du} \mathrm{VI}^{\mathrm{e}}$ siècle environ, comme celles du manuscrit 5111 de la British Library. L'invention de ce système de tables de concordances revient pourtant à Eusèbe de Césarée (265-339), au IV ${ }^{\mathrm{e}}$ siècle, comme en témoigne la lettre à Carpien, considérée comme le premier modèle. Le rôle de cette nouvelle mise en page est de faciliter la lecture de la liste des canons. Eusèbe reprend, approfondit et perfectionne le système mis en place par Amonius. Ce sont alors des listes séparées par deux, trois, quatre colonnes, selon les cas, que les enlumineurs choisissent de disposer pour y montrer les quatre évangiles, au moyen d'une arcature en plein cintre, en dégageant de la sorte une forme de tympan qui accueille, parfois, le numéro du canon - voir par exemple le manuscrit CITTÀ DEL vaticano, Biblioteca Apostolica, Vat. lat. 3803, fol. $2 \mathrm{v}^{\circ}$, IX siècle. On crée ainsi un espace de présentation efficace. L'articulation entre le fond et la forme est donc ici fondamentale et crée un système de liens forts entre les textes et l'image. Plusieurs exemples viennent illustrer ce fonctionnement organique, comme l'Évangile de Rabula FIRENZE, Biblioteca Medicea Laurenziana, Codex Pluteus, I, 56, vi siècle -, ou le Codex beneventanus - LoNDON, British Library, Add MS 5463, viII e siècle. Ces exemples font voir, cependant, une certaine diversité au sein d'un cadre unitaire, mais, dans tous les cas, les tables fonctionnent bel et bien comme une mise en relation interne entre les différents éléments. La mise en page est en même temps une mise en texte. La métaphore architecturale des tables est utilisée pour l'ensemble de l'ouvrage qu'elle structure et pour une meilleure visibilité/lisibilité grâce à une mise en valeur très claire des informations, en vue de l'effet produit sur le lecteur ${ }^{23}$.

L'époque de l'humanisme carolingien voit se consolider l'usage et la codification de ces tables de canons ${ }^{24}$, qui adoptent désormais une forme classique. C'est le cas de l'Évangéliaire de Lorsch, vers 780-820 - CITTÀ DEL VATICANO, Biblioteca, Pal. lat. 50 -, par exemple, dans lequel on remarque cependant quelques variations: la plastique architecturale, devenue carrée, et des copies de monnaies ou des représentations de bustes d'empereurs faisant directement référence à l'antique. D'autres indices, comme ici des paons, là des anges, peuvent être ajoutés, laissant apparaître les potentialités du champ de l'invention dans ce cadre pourtant contraint. Cette part d'invention va de pair avec les références à l'antiquité, tout en supportant les expressions directes d'une mode, voire plus en profondeur celles des aspirations d'une époque.

$\mathrm{Au} \mathrm{XII}^{\mathrm{e}}$ siècle, les tables des canons de la Bible de Saint-Bénigne de Dijon - DIJoN, Bibliothèque municipale, ms. 2, fol. $402 \mathrm{v}-405 \mathrm{v}$ - inscrivent sur leur support un phénomène tout à la fois d'appropriation et de réinterprétation important au sein même de l'ordre formel (fig. 4). 
Fig. 4 - DIJON, Bibliothèque municipale, ms. 2, fol. 402v (Canon de la Bible, Bible de Saint-Bénigne de Dijon, XII siècle) [source : enluminures.culture.fr].

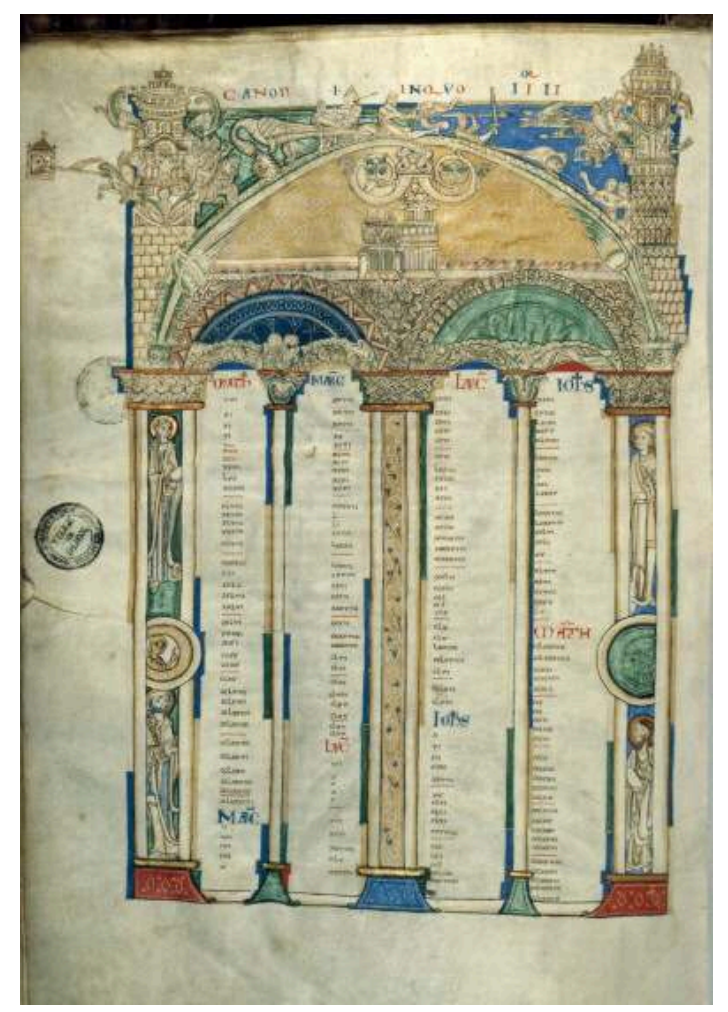

Un certain nombre des motifs du décor, devenu très riche, est inséré entre les feuillets, avec des portraits des évangélistes écrivant. La reprise - plus que la copie au sens littéral - n'empêche pas l'invention. Et les tables assurent toujours leur fonction de " raison graphique », au sens développé par Jack Goody : elles accentuent la valeur de document/monument, assurent une identité à l'instance reconnue, maitrisent les variances pour établir un « canon », ce qui accroît encore l'autorité de la chose écrite et présentée aux regards. La copie de la copie de la copie est l'affirmation d'une très forte continuité visuelle dans un espace hiérarchisé où le visuel prime sur le textuel ${ }^{25}$.

À la fin de la journée d'étude, la discussion a porté sur l'idée prégnante, tout au long des interventions, de la puissance créatrice de la copie qui permet un empilement de sens et une actualisation infinie du modèle. La copie n'est donc pas le degré le plus faible de la production, une dégradation sans cesse amplifiée, mais un acte de poièsis à part entière, de même que le modèle n'est jamais fermé. Il est en réalité un principe ouvert, et compris comme tel. Le rapport entre le modèle et la copie, loin d'être univoque, est en lui-même une production de sens, exactement comme il l'est dans les mathématiques. Est également revenue à plusieurs reprises la question de la reproduction actuelle, sous ses différentes formes et ses différents aspects photocopies, photographies, numérisation, fac-similés - qui témoignent tous d'une recherche de l'identique la plus précise possible, alors que la marge de différence, et donc d'invention, demeure intacte et que cette part de variance, de même que l'action de copier, révèlent toujours plus de nos habitudes de pensée et de vie en société.

Reçu : 11 novembre 2012 - Accepté : 14 novembre 2012 


\section{NOTES}

1. Pour exemple, voir F. MORA, « Mettre en roman ». Les romans d'Antiquité du XII siècle et leur postérité (XIII-XIV ${ }^{e}$ siècle), Paris, 2008, et la notion avancée de "technique allusive », qui rend compte de la dialectique entre le «modèle » et la modernité de la création, instituée par celui qui compose la matière du livre.

2. G. ORIGGi et F. DARBELlay (dir.), Repenser l'interdisciplinarité, Genève, 2010, en part. : D. PeSTRE, "L'évolution des champs de savoir, interdisciplinarité et valorisation », p. 39-56 et S. FULLER, «L'interdisciplinarité. La fin de l'image héroïque dans le marché des idées », p. 57-76; selon une double voie réflexive et critique, à partir d'une approche praxéologique et pragmatique, $\mathrm{V}$. HuYs et D. VERNANT, L'indisciplinaire de l'art, Paris, 2012.

3. P. R ÜCK, "Die Urkunde als Kunstwerk», in V.A. VON E UW et P.SCHREINER (dir.), Kaiserin Theophanu. Begegnung des Ostens und Westens um die Wende des ersten Jahrtausends. Gedenkschrift des Kölner Schnütgen-Museums zum 1000. Todesjahr der Kaiserin, t. 2, Cologne, 1991, p. 311-333 ; P. RÜcK (dir.), Graphische Symbole in mittelalterischen Urkunde. Beiträge zur diplomatischen Semiotik, Sigmaringen, 1996.

4. SEMUR-EN-AUXOIS, Bibliothèque municipale, ms. 1, fol. 71r-73r. Die Urkunden der Merowinger, éd. préparée par C. BRÜHL, publiée par T. KÖLZER, avec le concours de M. HARTMANN et A. STIELDORF, 2 vol., Hanovre, 2001 [MGH. Diplomata regum Francorum e stirpe merovingica], t. 1 ( $\mathrm{n}^{\circ}$ 3, p. 7-10; $\mathrm{n}^{\circ} 15$ p. 47-49) ; C. BRÜHL, "Clovis chez les faussaires", Bibliothèque de l'École des chartes, 154, 1996, p. 219-240, en part. p. 234-237 ; ID., Studien zu den merowingischen Königsurkunden, éd. T. KöLzER, Cologne/Weimar/Vienne, 1998, p. 75 sqq. ; A. RAUwEL, «La fausse charte de Clovis pour MoutiersSaint-Jean », Bulletin de l'Académie des sciences et des lettres de Semur, 2005, p. 66-71.

5. DIjon, Bibliothèque municipale, ms. 591, fol. 69v-70r. Die Urkunden der Merowinger..., op. cit, $\mathrm{n}^{\circ} 103$, p. 264-268. Voir K. HEIDECKER, « Le cartulaire et la chronique de l'abbaye Saint-Bénigne de Dijon : le manuscrit 591 de la Bibliothèque municipale de Dijon ", à paraître dans E. MAGNANI et M.J. GASSE-GRANDJEAN (dir.), Productions, remplois, mises en registre : la pratique sociale de l'écrit à travers la documentation médiévale bourguignonne.

6. Mâcon, Archives départementales de la Saône-et-Loire, H 675. Voir O. BRUAND, Les origines de la société féodale. L'exemple de l'Autunois (France, Bourgogne), Dijon, 2009, p. 18-19; I. FEES, «Drei Urkunden des Bischofs Jonas von Autun und die (angebliche) Synode von Saint-Geosmes", Deutsches Archiv für Erforschung des Mittelalters, 51/2 (1995), p. 376-403 ; C. BRÜHL, «Die Urkunde Karls des Einfältigen und Rudolf von Westfranken für das Nonnenkloster Saint-Andoche zu Autun ", in ID., Aus Mittelalter und Diplomatik, Gesammelte Aufsätze 2, Hildesheim/Munich/Zurich, 1989, p. 838-850, repris de «Eine Fälschung auf den Namen Karls des Einfältigen für Nonnenkloster Saint-Andoche zu Autun», Historisches Jahrbuch, 91 (1971), p. 384-393. L'expression « mini-cartulaire » est de Nathalie Verpeaux (Saint-Andoche et Saint-Jean-le-Grand : des religieuses à Autun au Moyen Âge, thèse de doctorat de l'université Paris 1 Panthéon-Sorbonne, 2009).

7. PARIS, BnF, nal 1497, 1498 et 2262. Voir H. ATSMA et J. VEZIN, « Gestion de la mémoire à l'époque de saint Hugues (1049-1109) : la genèse paléographique et codicologique du plus ancien cartulaire de l'abbaye de Cluny", Histoire et archives, 7 (2000), p. 5-29; ID., "Originaux et copies: la reproduction des éléments graphiques des actes des $10^{\mathrm{e}}$ et $11^{\mathrm{e}}$ siècles dans le cartulaire de Cluny ", in А. козто, А. WinRoтн (dir.), Charters, Cartularies and Archives, Rome, 2002, p. 113-128; S. BARRET, La mémoire et l'écrit: l'abbaye de Cluny et ses archives ( $X^{e}$-XVIII ${ }^{e}$ siècle), Münster, 2004, p. 247-287 ; M. HILLEBRANDT, "Les cartulaires de l'abbaye de Cluny », Mémoires de la société pour l'histoire du droit et des institutions des anciens pays bourguignons, comtois et romands, 50 (1993), 
p. 7-18; D. IOGNA-PRAT, «La confection des cartulaires et l'historiographie à Cluny $\left(\mathrm{XI}^{\mathrm{e}}-\mathrm{XII}^{\mathrm{e}}\right.$ siècles) ", in O. GUYOTJEANNIN, L. MORELLE et M. PARISSE (dir.), Les Cartulaires. Actes de la Table ronde..., Paris, 1993, p. 27-44; ID., «La geste des origines dans l'historiographie clunisienne des $\mathrm{XI}^{\mathrm{e}}$ et $\mathrm{XII}^{\mathrm{e}}$ siècles ", Revue bénédictine, 102 (1992), p. 135-191, ici p.153-170 (nouv. éd. dans ID., Études clunisiennes, Paris, 2002, p. 161-200); B. H. ROSENwEIN, «Cluny's immunities inthe Tenth and Eleventh Centuries. Images and narratives ", in G. CONSTABLE, G. MELVILLE et J. OBERSTE (dir.), Die Cluniazenser in ihrem politisch-sozialen Umfeld, Münster, 1998, p. 133-163, ici p. 145-163 ; M. SCHAPIRO, The Parma Ildefonsus, a romanesque illuminated manuscript from Cluny and related works. S. 1., College Art Association of America, 1964.

8. Voir, sur la problématique énoncée, l'enquête collective conduite par É. JoLLET et C. MASSU, sous l'intitulé Les images du monument de la Renaissance à nos jours, Rennes, 2012, pour une approche renouvelée de ce qui constitue la mémoire collective dans l'espace public et ce que recouvre le monument ou son dessin, son esquisse, son graphe.

9. Sur toutes ces perspectives d'études, A. RITZ-GUIBERT, «La collection Gaignières : méthodes et finalités ", Bulletin monumental, 166/4 (2008), p.315-338; à partir de l'exemple particulier de support que sont les sceaux, suivant la même problématique, $E A D$., «Les sceaux médiévaux au XVII ${ }^{\mathrm{e}}$ siècle : les dessins de sceaux dans la collection Gaignières (1642-1715) », in M. G IL et J.L. CHASSEL (éd.), Pourquoi les sceaux? La sigillographie, nouvel enjeu de l'histoire de l'art, Lille, 2011, p. $45-60$.

10. M. BEAUjour, Miroirs d'encre, Rhétorique de l'autoportrait, Paris, 1980.

11. Sur ce processus, à partir d'analyses de l'art le plus contemporain, R. RECHT, Point de fuite. Les images des images des images. Essais critiques sur l'art actuel, 1987-2007, Paris, 2009, en part. $1^{\text {re }}$ partie, p. 17-74, «Critique et histoire de l'art ». À partir d'exemples médiévaux, H. BELTing, Image et culte. Une histoire de l'art avant l'époque de l'art, trad. fr. F. MULLER, Paris, 1998 (Berlin, 1990).

12. Sur la distinction, très souple, très poreuse, aux premiers siècles du christianisme, entre évangiles apocryphes et évangiles canoniques, H. KOESTER et F. B ovon, Genèse de l'Écriture chrétienne, Turnhout, 1991, en part. p. 59-106.

13. J.-C. Sснмітт, La raison des gestes dans l'Occident médiéval, Paris, 1990, en part. p. 25-26; voir aussi, ID., Le corps des images. Essais sur la culture visuelle au Moyen Âge, Paris, 2002.

14. F. GARNIER, Le langage de l'image au Moyen Âge. Signification et symbolique, Paris, 1982², p. 223.

15. A. VAN DIJK, « The angelic salutation in early byzantine and medieval Annunciation imagery », The Art Bulletin, 81/3 (1999), p. 420-436.

16. V. SCHIER, "Memorials sung and unsung : liturgical remembrance and its history ", in Care for the Here and the Hereafter: Memoria, art and ritual in the Middle Ages, Turnhout, 2005, p. 125.

17. Pour un élargissement de ces problématiques, en particulier aux arts non-occidentaux et à leurs modes de réception, voir C. SEVERI, Le principe de la chimère. Une anthropologie de la mémoire, Paris, 2007, attentif aux «supports mnémoniques » dont la forme mobilise le regard et invite à les décrypter.

18. Pour une double situation, historique et iconographique, des rôles désormais dévolus aux anges, entre XIV ${ }^{e}$ et $\mathrm{XV}^{\mathrm{e}}$ siècles, au Puy-en-Velay, en Auvergne toujours, H. MILLET et C. RABEL, avec une contribution de B. Motrin, La Vierge au Manteau du Puy-en-Velay, Lyon, 2011.

19. C. F. HARDY, "The music in the glass of the Beauchamp Chapel at Warwick », Sammelbände des Internationalen Musikgesellschaft, 3/3 (1902), p. 454-467; L. MONCKTON, «Fit for a king? The architecture of the Beauchamp Chapel », Architectural History, 47 (2004), p. 25-52.

20. Deux exemples: le De institutione canonicorum (psaume «Je te chante en présence des anges ») ; la Doctrina de Jean Gerson qualifiant les anges d'« images de la cour céleste ».

21. À présent, pour une démonstration d'ensemble, voir M.C HARBONNEL, Materialibus ad immaterrialia. Peinture murale et piété dans les anciens diocèses de Clermont, du Puy et de Saint-Flour du 
$\mathrm{XII}^{e}$ au XVe siècle, thèse de doctorat sou la direction de B. Phalip et D. Russo, université Blaise Pascal Clermont-Ferrand II, 2012.

22. M.-C. PEREIRA, «Les tables de canons: la Bible de Saint-Bénigne. Notes pour un projet de recherches en cours", Bucema, 12 (2008), p. 299-301 [http://cem.revues.org/index7172.html]. C'est dans un cadre problématique large des rapports entre contenant/contenu, imaginaire/ image, que l'auteur s'est intéressée à cet exemple peu commenté de la Bible de Saint-Bénigne de Dijon, manuscrit 2 de la bibliothèque municipale de Dijon. Sur ce manuscrit, Y. ZALUSKA, L'enluminure et le Scriptorium de Cîteaux au XII ${ }^{e}$ siècle, Cîteaux, 1989, p. 144-147, notice 88, p. 262-263, et annexe II, p.276-277; EAD., collab. M-.F. DAMONGEOT, F. SAULNIER et G. L ANÖ̈, Manuscrits enluminés de Dijon, Paris, 1991 (Corpus des manuscrits enluminés des collections publiques des départements).

23. Outre les travaux de J. Goody (1919), parmi lesquels La raison graphique et la domestication de la pensée sauvage, trad. fr. J. BAZIN et A. BENSA, Paris, 1978 (Cambridge, 1977), pour les effets visuels cognitifs de la transcription, voir T. IMGoLD, Une brève histoire des lignes, Paris, 2011. Voir aussi I. SERÇA, Esthétique de la ponctuation, Paris, 2012, en part. p. 278-289, « Donner une forme au temps. L'hypogée des deux points ".

24. E. DE BRUYNE (1898-1959), Études d'esthétique médiévale, Paris, 1998 (Bruges, 1946), t. 1, livre II, «La civilisation carolingienne », p.163-370, est en ce sens d'actualité. Pour un ensemble de réflexions amples sur ce champ d'interrogation qui demeure ouvert, voir M. LEMoINE, Scriptora minora, Paris, 2012 (Cahiers de recherches médiévales et humanistes, 23).

25. Pour un autre contexte, dans un autre environnement, J. ASSMAN (1938), La mémoire culturelle. Écriture, souvenir et imaginaire politique dans les civilisations antiques, trad. fr. D. MEUR, Paris, 2010, (Munich, 2002), sur la «mémoire communicationnelle » et la "mémoire culturelle ", ainsi que sur « le principe consacrant » alors à l'œuvre, en part. p. 79-116 (« La culture de l'écrit »).

\section{INDEX}

Mots-clés : anthropologie, histoire de l'art 